\title{
Animal Research Support: The Transition from Ancillary Service to Contract Research Organization
}

\author{
Jerry Zamzow, Assistant Vice President for Research \\ Iowa State University
}

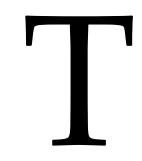

he use of animals in research is a vital component in the formula for advancing the human (and animal) condition. The use of animals in research is, at times, controversial. Engaging in these activities should not be taken lightly given the lightning rod that this work can be. Public perception and understanding has shifted over the past few decades, and institutions assume much responsibility, along with significant amounts of risk when working in the sphere as we strive to advance science and knowledge. Centralizing more of this responsibility and risk is a mechanism to ensure not only the regulatory aspects of this work are met to the fullest extent, but also can be used to mitigate questionable research practices and reduce bias.

\section{Historical Aspects of Animal Re- search}

Though animals are used in a wide array of disciplines, the greatest use has been in the life sciences sphere. Researchers have grown through systems that have a tradition of using animal models as part of their scientific inquiry. The majority of studies utilizing animals have historically been single investigator laboratories where the principal investigators provided much of the hands-on training for staff and students. Vivarium operations largely served to provide husbandry, medical care and appropriate space for the studies to be carried out. The regulations governing the use of animals in research and compliance thereof was comparatively manageable. A strong focus on animal welfare based on performance measures was the norm.

\section{The Shift}

As society expands its understanding of the world and biological systems associated with it, understandably, public perception of the use of animals in research has also shifted. The regulations and guidance documents from governing agencies have responded to this shift as well; growing in sheer volume along with prescriptiveness in terms of engineering standards and the expectations that users of animal models are also versed in knowledge and application of the requirements. Along with written regulatory pressure, there has also been increased scrutiny from those agencies charged with upholding the regulations, along with voluntary accreditation bodies whose stamp of approval serve as a gold standard for robust animal care and use programs. That is not to say that this shift is a negative trend for animal research. I would posit that the shift is indeed a positive one in that it ensures that institutions and individual researchers are being deliberate in their approach to the matter. This trajectory, nonetheless, does pose greater risks for an institution that must be addressed and mitigated.

Along with the shift in compliance aspects, the science itself has shifted. The rise of interdisciplinary research has brought non-traditional animal users into 
the space. Further, the studies that are being carried out are much more advanced in nature. Advances in in vitro and virtual realms have allowed for a reduction in the use of animals in preliminary work. This is a positive advancement, and rings true to the three paramount tenets of the use of animals in research: Reduce, Refine, and Replace-the "3Rs". With these advances also come challenges. As the traditional "feed them and weigh them" studies at agricultural schools are now more the exception rather than the rule, the procedures to be carried out in the models are much more advanced, intense, and prescript. This brings with it a need for greater inputs on training of researchers and their staff, along with advanced space, equipment and overall expertise.

What could a CRO look like?

To help researchers overcome what may be perceived as barriers to using animal models, an operational model that might be employed is for an institution's vivarium to function as a contract research organization (CRO) rather than an ancillary service. The vision would be that the vivarium functions as a "one stop shop" for investigators. The CRO would function like commercial CROs currently do by providing assistance in study design, animal use and standard operating procedure development, carrying out the activities, and subsequently returning the results to the investigative team.

What are the pros to this model?

Benefits to this model are potentially many. Institutional risk is mitigated by having highly trained staff being intimately involved with all aspects of a study. Reducing protocol drift and adverse events that may occur due to human error associated with unfamiliarity of techniques and procedures might also increase the reliability in results and reduce unnecessary repetition of studies due to failures; again helping achieve the goals of the aforementioned "3Rs".

Research is an ever-changing and sometimes unpredictable endeavor, especially in biological systems. As the research unfolds, naturally investigators seek to follow the new path that they have been put on. At times, this may lead to questionable research practices that put the investigator and institution at risk. By managing the process in a controlled and unbiased environment, this fluidity can be managed to ensure that the studies are carried out with a greater confidence in regulatory and ethical integrity.

Functional and operation benefits include cost-savings to be realized for focused training efforts (i.e., a core group versus an entire institution), non-duplication of efforts and equipment, and less taxing of other systems (i.e., the IACUC). Additionally, institutions will likely garner a greater sense of confidence in their animal care and use program due to more controlled environments as the studies are carried out. Further, a more efficient use of space might be realized as the cyclical availability of researchers having students and staff to carry out the studies would be eliminated. Most importantly, principal investigator time savings will be realized through a reduction in administrative burdens and the actual time spent conducting the experiments. 


\section{What are the cons and changes needed?}

As the duties of carrying out the animal studies shift to other parties, perhaps the greatest negatives to this model are the loss of a teaching opportunity and a sense of closeness to the work. Given the shift in types and disciplines that many animal studies are stemming from, the culture of animal use is not engrained, perhaps rendering this moot. Additionally, a negative would be the up-front financial costs associated with the model. Having sufficient personnel and associated expertise, along with the physical infrastructure related to equipment and space does not come without cost. While direct dollars spent may increase, the previously mentioned savings will likely offset this.

Changes needed to operationalize such a model most importantly include central support. An investment in the model would be needed to make it functional, with the realization that costs may not be recouped for some time. However, with proper changes to cost structures and fee schedules, such a program would likely become self-sustaining.

Perhaps the most significant change needed would be to the institution's human capital. While traditional animal caretakers will always be imperative to these operations, skilled individuals not only trained in functional technique, but also scientific processes will be needed to make the CRO successful. Providing a robust and continuous training program for these individuals is a lynchpin for success of the model. Failure to do so will likely result in the loss of the many benefits.

\section{Conclusion}

In sum, the model of a CRO is an opportunity for an institution and research offices to provide a greater level of service to today's investigators and their research. As researchers face many pressures and challenges, this is a mechanism to aid in minimizing those, while also assisting the institution in addressing the pressures and challenges it faces. 